\title{
- La armadura mudéjar del Palacio de los Marqueses de Moctezuma en Ronda (Málaga)
}

\author{
Rafael Valentín López Flores
}

No seremos nosotros los que a estas alturas demos nuevas pautas o ampliemos en algún aspecto los magníficos estudios de lo mudéjar en Málaga, pero si nos vemos en la obligación de comunicar la existencia de una nueva armadura de éste estilo en la ciudad de Ronda -hasta ahora nunca mostrada-, para completar así el catálogo de éstas en la provincia.

En Ronda se conocen varios ejemplos de armaduras mudéjares, algunas conservadas, otras tristemente desaparecidas, y hasta algunas ocultas, que han fijado de manera notable esta manifestación artística en la localidad. La mayoría de ellas se fechan en el siglo XVI, y se relacionan con espacios religiosos o aristocráticos; existiendo varias tipologías.

Del tipo de par y nudillo existió una armadura cubriendo la nave de la iglesia del convento de San Francisco, que tras sufrir graves daños durante la Guerra de Independencia despareció definitivamente en 1936'. Del tipo denominado de aljarfe (armaduras planas sin decoración de lazo) se conserva una pequeña franja cubriendo la galería superior del patio mudéjar del Palacio de Mondragón y el techo de la ropería del Convento de Santa Isabel de los Ángeles; decorado mediante técnicas ataujeradas en sus jácelas con dobles lazos de ocho y punteados blancos en el fondo de sus tabicas ${ }^{2}$. También existe un verdadero tajuel (armadura plana decorada por lazo) en la Iglesia de Padre Jesús (antigua de Santa Cecilia), bajo su coro, formado por crucetas y estrellas de lazo de cuatro ${ }^{3}$. La torre de la Colegiata de Santa María de la Encarnación la Mayor también conserva una armadura, ochavada, con almizate decorado mediante estrellas de ocho puntas y crucetas, que a su vez decoran la parte baja de los faldones ${ }^{4}$.

Entre las desaparecidas u ocultas tenemos la que cubría la Iglesia de la Virgen de la Paz, que según fotografía del malagueño Archivo Temboury era una armadura de lazo bicolor, decorada mediante crucetas y estrellas con rosetas pintadas asemejando soles en su interior. La que cubría la nave del Convento de la Merced únicamente ha conservado el arranque de un tirante sobre el coro actual. De la

LÓPEZ FLORES, Rafael: "La armadura mudéjar del Palacio de los Marqueses de Moctezuma en Ronda (Málaga)", en Boletín de Arte ${ }^{\circ}$ 25, Universidad de Málaga, 2004, págs. 783-794. 
posiblemente oculta del Convento de Clarisas del Patrocinio de San José - de nuevo sólo conocida gracias a fotografías del Archivo Temboury - sabemos que era del tipo de limabordón, con decoración de lazo formando crucetas y estrellas, y almizate y tirantes realizados en maderas policromas. Respecto a la del desaparecido Hospital Real de Santa Bárbara, conocemos de la existencia, antes de su demolición, de una sola viga tirante oculta sobre el techo de su capilla; y de la también desaparecida del que fuera antiguo Ayuntamiento, que según D. Juan Temboury tenía un almizate decorado por estrellas de las que pendían adornos colgantes a modo de estalactitas. ${ }^{5}$

Dejamos para el final las conocidas como de limamohamar y limabordón, que al igual que las anteriores hemos de datar en el trascurso del siglo XVI. Del primer tipo es la que cubre el salón de la casa rondeña de los Sres. Boyd, considerada por los estudiosos de la materia como una de las que atesora mayor calidad e interés de la provincia; cubierta por una raro lazo de diez y con dos tirantes dobles sobre zapatas como apoyo. ${ }^{6}$ De limabordón se conservan dos importantes ejemplos que guardaran relación estilística con la del Palacio de los Marqueses de Moctezuma. La primera es la que cubre el salón principal del Palacio de Mondragón, con un primer tramo plano decorado por casetones y sustentado por gruesas zapatas renacentistas, y el resto configurado por una armadura de limabordón de pares y jaldetas gramillazas, que decora su almizate con lazo de ocho formando crucetas y estrellas cuyas tabicas se decoran con rosetas pintadas a modo de soles. La segunda armadura es la de la iglesia del ex-Convento de Santo Domingo, que se cree decoraba su almizate y faldones a base de lazo formando crucetas y estrellas de ocho puntas formando tres calles; el resto lo hace mediante tablas almenadas pintadas con rosetas y escudos dominicos, jaldetas y pares abilletados con perlas y ovas en tinta oscura, tirantes a base de azafates y crucetas con sus perfiles decorados por rombos polícromos, y otros elementos diversos donde se repiten los escudos, las decoraciones vegetales o las palmas, ejecutados mediante técnicas similares a la taracea.

Una vez repasados los ejemplos conocidos, tenemos necesariamente que pasar a la que verdaderamente nos ocupa, la del Palacio de los Marqueses de Moctezuma, y para ello vemos necesario aludir primero a la casa-palacio que la alberga.

El Palacio de los Marqueses de Moctezuma se encuentra levantado en el barrio más antiguo de Ronda, conocido como La Ciudad, habitado desde tiempos

\footnotetext{
${ }^{1}$ AGUILAR, Marfa Dolores: Málaga Mudéjar. Arquitectura religiosa y civil, Málaga, Universidad, 1979, pág. 68.

${ }^{2}$ Ibídem; págs. 184 y 164.

${ }^{3}$ Ibídem; pág. 185.

${ }^{4}$ Ibídem; pág. 182.

${ }^{5}$ AGULLAR, María Dolores: op. cit., pág. 197-198.

${ }^{6}$ Ibídem; págs. 174-175.

${ }^{7}$ Ibídem; págs. 171-172.
} 


Q: Varba

protohistóricos. La familia que erigió el palacio -al menos tal y como hoy lo conocemos-, desciende del que fuera mítico y noveno emperador azteca, y tiene sus primeros descendientes conocidos en Ronda en las personas de Diego, Pedro y Pedro Manuel Moctezuma Loaysa ${ }^{8}$-caballeros de la Real Maestranza de Caballería de Ronda desde 1707-. Durante el siglo XVIII destacó en la familia D. José Moctezuma y Rojas, nieto por línea directa del mítico emperador cuyo panteón se conserva en la capilla del Rosario del rondeño Convento de Santo Domingo; ${ }^{9}$ ya en el siglo XIX Doña María Teresa Holgado Vázquez de Mondragón y Moctezuma, última descendiente de los Moctezuma rondeños nacida en dicha ciudad en 1824 , que a su muerte en 1865 legó en testamento la primera cantidad de dinero que hizo falta para fundar el Monte de Piedad de Ronda. ${ }^{10}$ Posteriormente Caja de Ahorros de Ronda, y hoy parte de Unicaja es la propietaria del edificio, donde ha dado cabida al Museo Joaquín Peinado

Los Moctezuma -una vez vistas las palabras que preceden a éstas- debieron llegar a Ronda a finales del siglo XVII, y erigieron su casa palaciega en la Ciudad, justo en la que hoy se conoce como Plaza del Gigante. Dicho palacio, que sin duda se levantó sobre construcciones precedentes de estilo musulmán o renacentista, se adapta al trazado sinuoso propio de la que fue medina musulmana, y presenta rasgos estilísticos que lo sitúan como obra del siglo XIX, aunque con elementos interiores de siglos anteriores; siendo el principal de estos la armadura mudéjar. La estructura exterior y elementos destacados del palacio se explican perfectamente en las palabras de Emilia Garrido Oliver:

".es una composición dividida en tres pisos cuyos huecos, comenzando por la derecha, siguen ejes verticales; en los primeros, y a partir del tercero, se advierte la ausencia de vanos en el piso bajo; tan sólo uno, correspondiente al cuarto eje, volverá a repetir el esquema. Ello se explica, por la estrechez de la calle, y por la existencia de dos patios en el interior de la vivienda, que solucionaba el problema de la iluminación y ventilación de esta zona de la planta baja.

La portada principal, que queda resaltada del conjunto, por su tonalidad diferente, su decoración, heráldica y floral, y por el balcón principal -colocado justo encima- se convierte en la protagonista del conjunto. Su estructura adintelada permite reservar el dintel para los escudos, mientras que las jambas se decoran con pilastras planas sobre plinto y alargadas con trozos de

\footnotetext{
s GARRIDO DOMÍNGUEZ, Antonio: "Una familia rondeña: Los Moctezuma", en AA. VV: Miscelánea de estudios rondeños y otros estudios. Homenaje al profesor don Manuel Martín Rivero, Málaga, Caja de Ahorros de Ronda, 1981, págs. 317-330.

${ }^{9}$ MORETI, Juan José: Historia de la muy noble y muy leal ciudad de Ronda, Ronda, Establecimiento tipográfico del autor, 1867, pág. 848.

${ }^{10}$ GARRIDO DOMÍNGUEZ, Antonio: op. cit., págs. 324-330.
} 


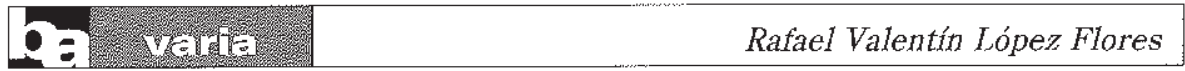

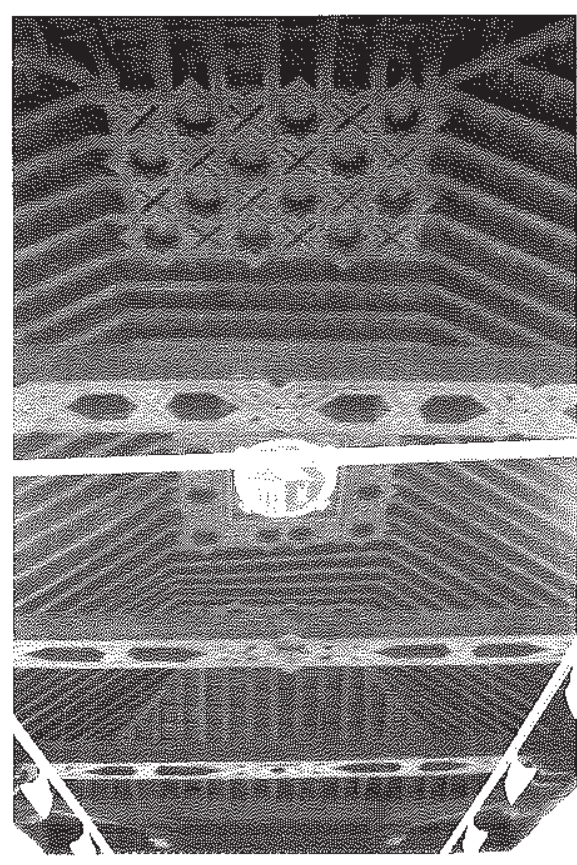

1. Vista general de la Armadura del Palacio de los Marqueses de Moctezuma, Ronda

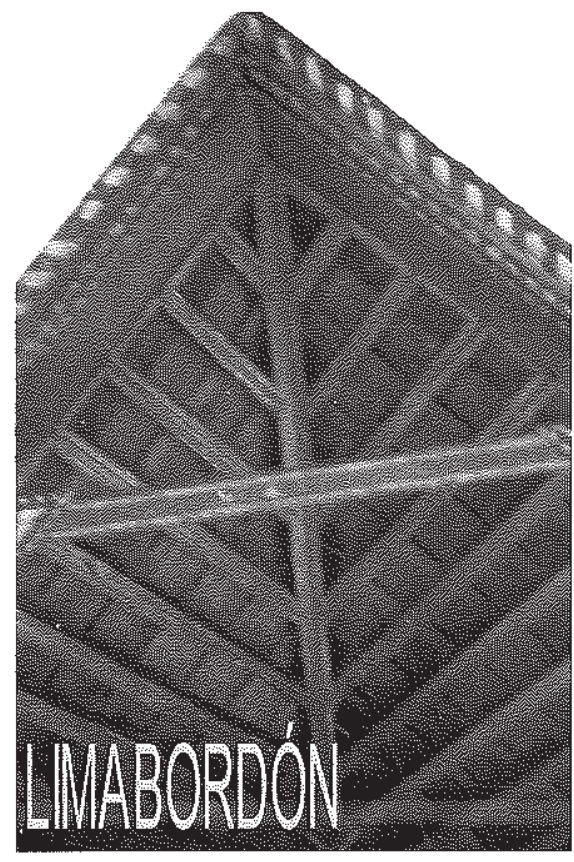

2. Armadura del Palacio de los Marqueses de Moctezuma; detalle de uno de sus vértices

entablamento, que se adornan con motivos decorativos vegetales muy esquematizados, encajados en dos fajas rectangulares que recorren prácticamente todo su fuste. ${ }^{+1}$

La armadura se sitúa en el salón principal del palacio, en su segunda planta, y cubre un espacio rectangular de aproximadamente 5 metros en sus lados cortos y 15 metros en los largos. Hemos de catalogarla dentro de las armaduras rectangulares de lazo denominadas de limabordón (FiG. 1), dado que es solamente una viga o lima la que sirve de unión o transición entre los faldones menores y mayores (FiG. 2).

\footnotetext{
${ }^{11}$ GARRIDO OEIVER, Emilia: "El palacio de los Marqueses de Moctezuma", en Guía Museo Peinado, Ronda, Fundación Ưnicaja Ronda, 2003, s. p.
} 


\section{Armadura del Palacio de los Marqueses de Moctezuma; argeture $y$ almarbate}

\section{Armadura de1 Palacio de 1 os Marqueses de Moctezuma; vista de uno de sus cuadrales}
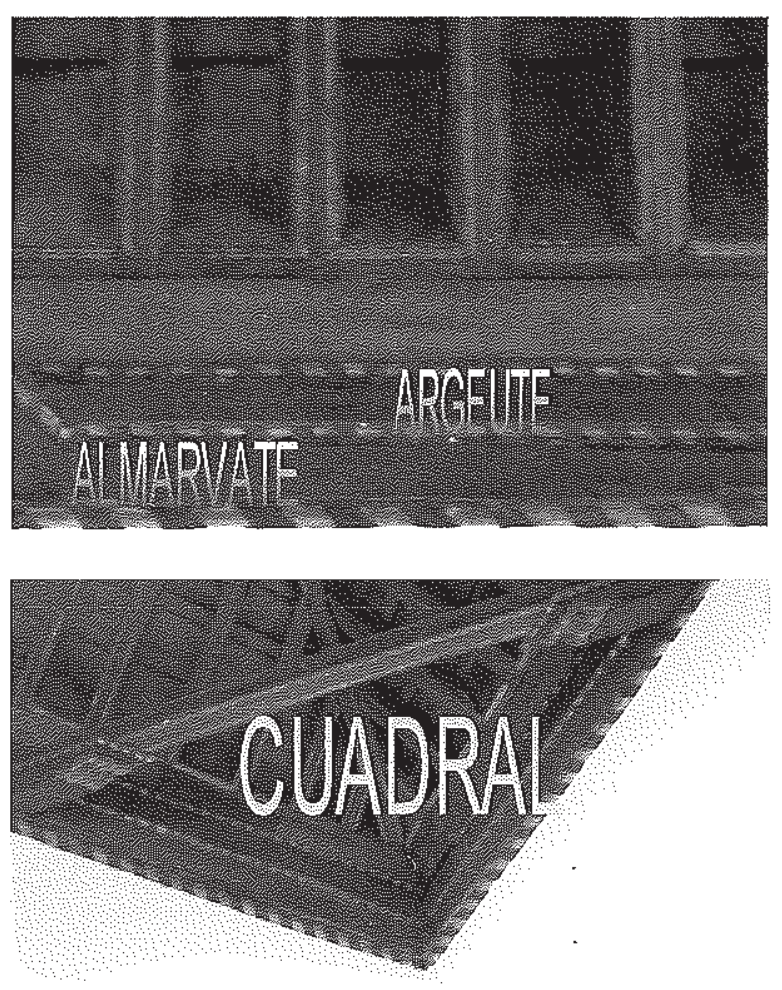

Si empezamos a describir la armadura en nivel ascendente diremos que se sustenta sobre almarvate (estribo sobre el muro del salón) y argeute (franja superior de este primer elemento a modo de entablamento que da altura a la armadura) (Fic. 3) que recorren todo su perímetro, decorados por tres molduras convexas abilletadas, de las que la inferior resalta su condición de unión entre la armadura y el muro mediante su mayor anchura, mientras que las superiores, que sirven para enmarcar el argeute, son de igual anchura y siguen la misma decoración que la inferior mediante cortes oblicuos acompasados sobre la moldura, que se realzan decorativamente mediante su dorado, en contraste con el color oscuro de la madera con la que está realizada la armadura.

Sobre el almarvate o estribo descansan tres tirantes y cuatro cuadrales que cohesionan la estructura de la armadura. Los cuadrales (Fis. 4), situados en las esquinas de la armadura, se sustentan por su parte inferior por zapatas rematadas en sus cabezas por triple moldura con perfil en $S$, muy del gusto renacentista (FrG. 5), resaltadas de nuevo respecto al resto de la armadura mediante su dorado, y desarrollando en todo su perfil superior la moldura abilletada que corre por el inicio 


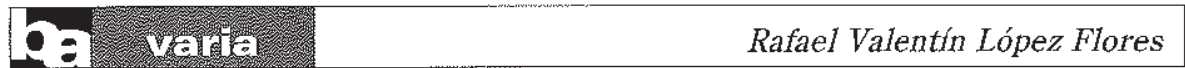

5. Armadura del Palacio de los Marqueses de Moctezuma; zapata de uno de sus cuadrales
6. Armadura del Palacio de los Marqueses de Moctezuma; uno de los faldones menores y sus cuadrales
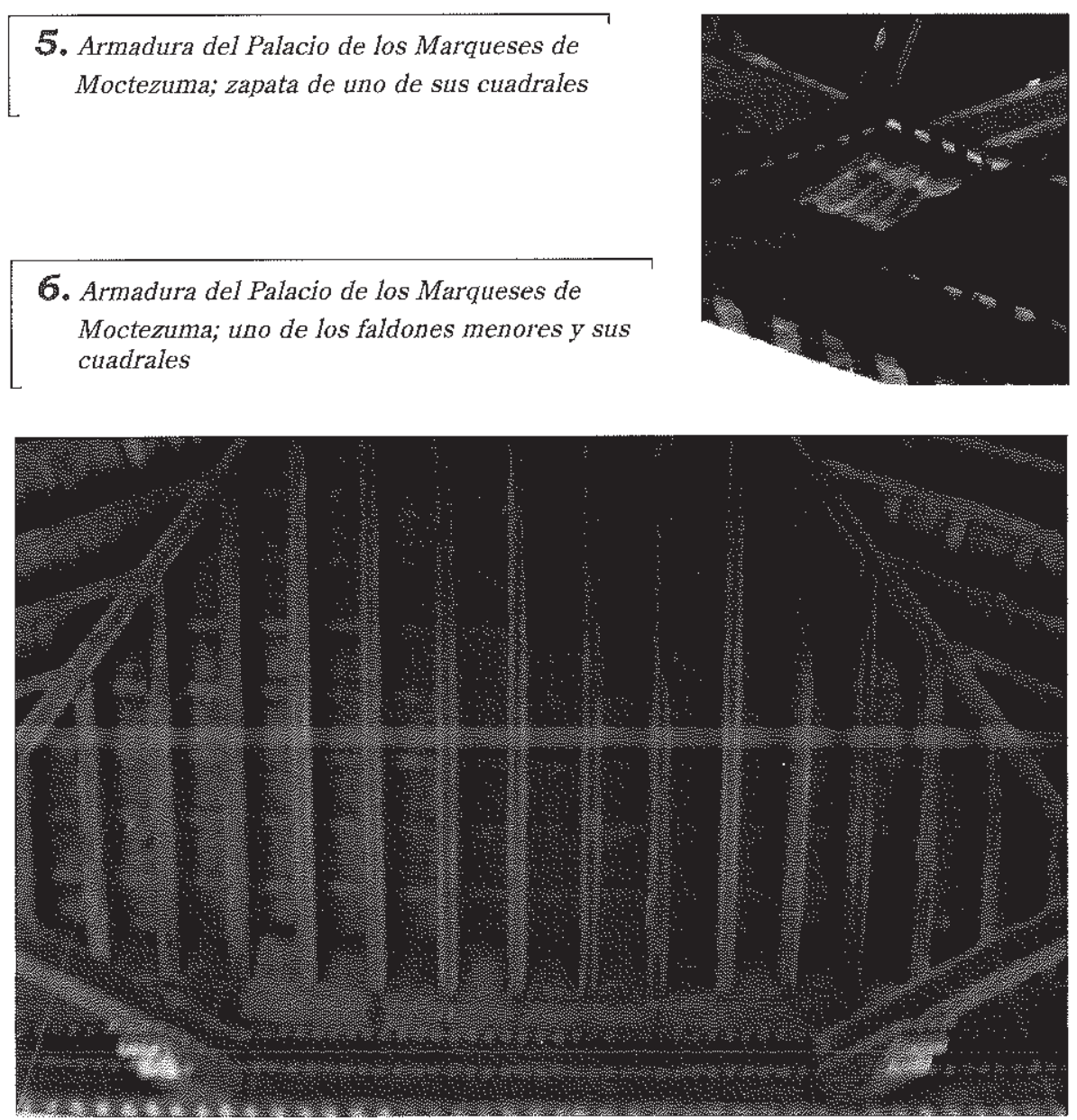

del argeute. El cuadral en sí, solo presenta como decoración dos pequeñas molduras enteramente doradas en su cara inferior; molduras que como norma general decoraran todos los perfiles de la armadura (F/G, 6).

Los tres tirantes dobles de la armadura presentan la misma anchura y los mismos elementos de sustentación; unos canes dobles rematados en sus cabezas por molduras doradas de tipo renacentista con doble perfil en $S$ (F/G. 7), que también desarrollan la moldura del argeute como en el caso de las zapatas de los cuadrales. Presentan una decoración muy similar en todos los casos, a base de crucetas, estrellas y azafates con sus perfiles exteriores e interiores dorados, pero difieren en la disposición y formas geométricas concretas que éstos adoptan. 


Q. Varmadura mudéjar del Palacio de los Marqueses...

\section{Armadura del Palacio de los Marqueses de Moctezuma; extremo de una de las vigas tirantes con sus canes}

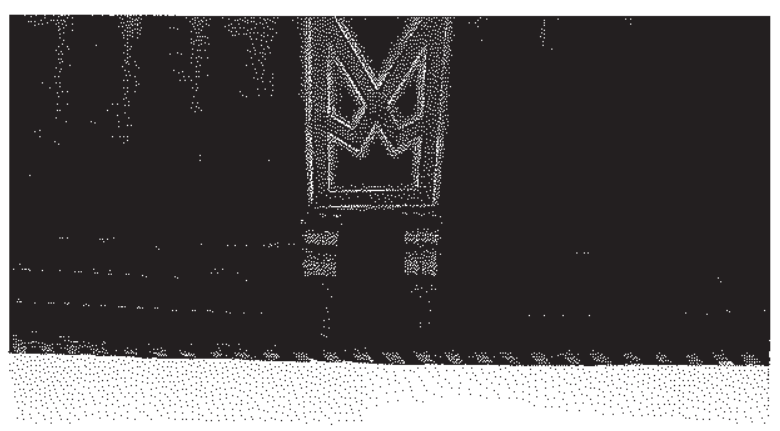

\section{Arnadura de1 \\ Palacio de los \\ Marqueses de Moctezuma; tirante de los pies de la armadura}

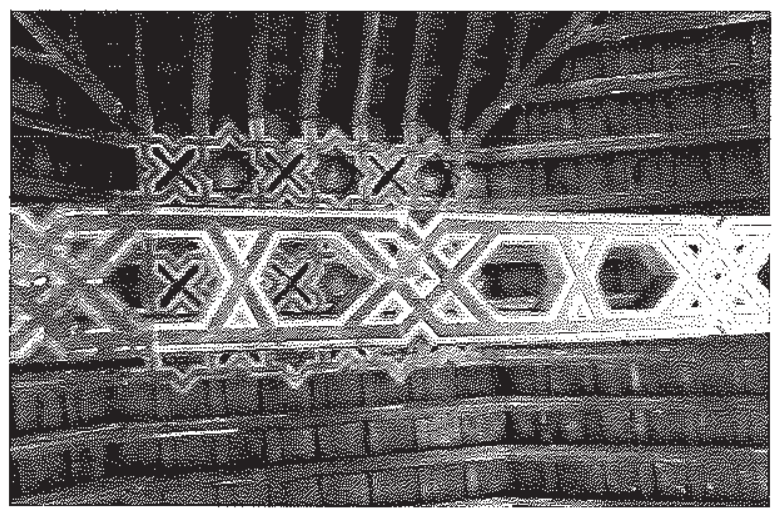

El primero de ellos (F/G. 8) -situado a los pies de la armadura, sí consideramos como referencia la puerta de entrada al salón como referencia- se decora mediante crucetas y azafates calados, repitiendo un diseño que presenta en su centro una cruceta que se repetirá hasta tres veces en intervalos regulares, interponiendo entre éstas dos figuras trapezoidales de seis lados que en cercanía de las crucetas cierran sus ángulos para acercarse al centro geométrico de éstas. Entre estas crucetas se disponen otras menos elaboradas, realizadas a base de pequeños triángulos equiláteros (Fic. 9). Como peculiaridad, este tirante presenta sus perfiles decorados a base de pequeñas puntas de diamante hacia su interior, situadas en el centro de cada una de las crucetas.

Si la decoración de los tres tirantes es diferente en cada uno de los casos como norma general, sí que ahí un elemento que los hace homogéneos; sus remates junto a los canes que los sustentan (FiG. 7). Estos remates se forman en los tres casos mediante el uso del mismo número de figuras geométricas, de las cuales, la más cercana a los canes es un azafate de forma trapezoidal -constituido por un pequeño rectángulo que en su parte central, abierta al tirante, elabora un remate triangular en punta dentada a modo de diamante, y unos medios triángulos a los lados-, siendo las dos restantes -enlazadas a dicho azafate- dos pequeñas figuras romboides que guardan perfectamente la simetría decorativa impuesta por el motivo anterior. 


Q: Varia $\quad$ Rafael Valentín López Flores
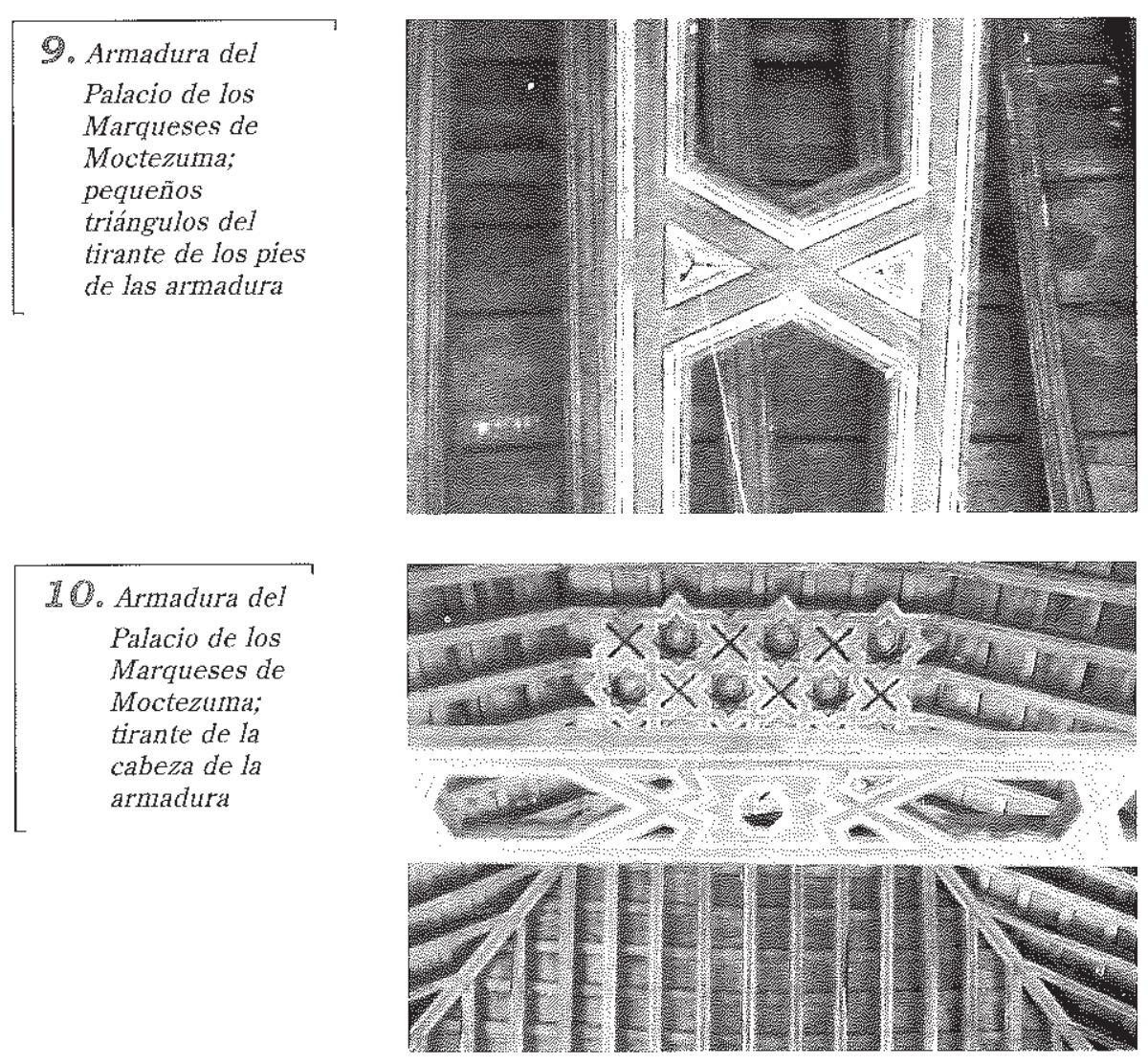

El tirante de cabeza (FiG. 10), situado justo al final de la estancia, repite respecto al anterior el esquema geométrico de sus extremos; pero cambia su decoración central y los perfiles en puntas de diamante hacía su interior, ya que al perder las crucetas se harían poco coherentes. De esa manera presenta en su centro un octógono enmarcado por un rectángulo, rematado en sus lados cortos por el mismo tipo de dibujo que el presentado por las figuras cercanas a los canes, y realizando la transición decorativa hacia los trapecios irregulares por el mismo tipo de figura romboidal que ya hemos visto.

Por último, el tirante central se destaca del resto en tres de sus elementos decorativos (F/Gs. 11 × 12), todos ellos situados en la parte central del tirante, y guardando la proporcionalidad y simetría básicas en la decoración de la armadura. Aqui el centro lo ocupa ahora una estrella de ocho puntas -igual a las que después veremos en el almizate-, que en su centro, saliendo desde un círculo dorado, alberga una piña dorada que pende hace el suelo con una argolla metálica en su centro; desde donde seguramente pendería algún tipo de fámpara o similar que alumbraría 


\section{Armadura del Palacio de los Marqueses de Moctezuma; tirante central de la armadura}

la sala. La estrella prolonga una de sus puntas -justamente la que sigue el eje longitudinal de la armaduraen el perfil del tirante, para repetir la decoración en punta de diamante que vimos en el tirante de los pies, pero ahora hacia fuera, y sólo en el centro del tirante. Su última variación decorativa la constituyen los motivos geométricos que engarzan por ambos lados a la estrella de ocho puntas, que

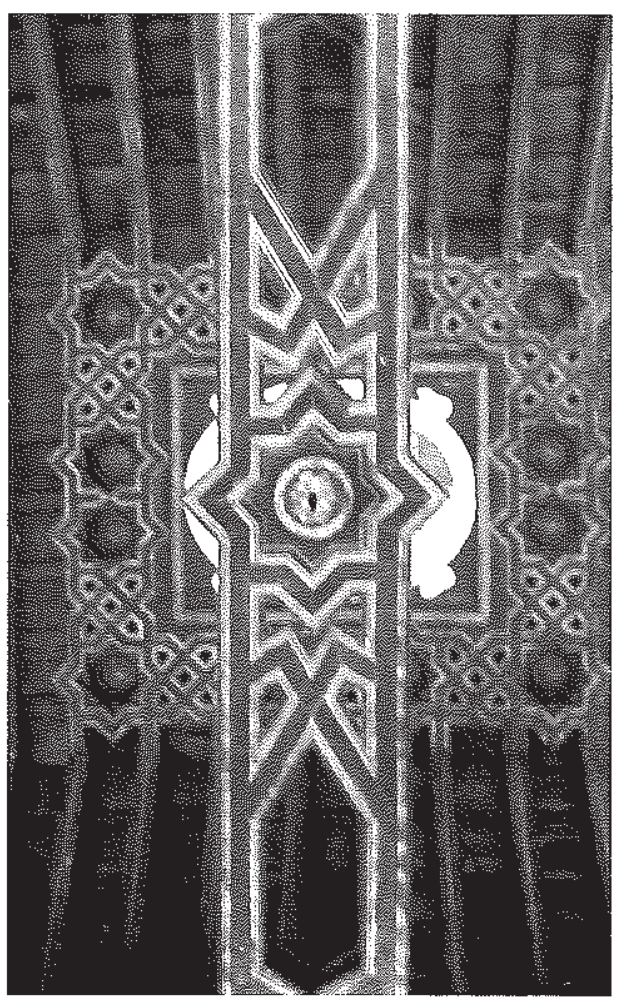
adoptan el mismo esquema en punta que vimos respecto a los motivos cercanos a los canes; pero dobles, suprimiendo de ese modo el lado plano de los mismos.

La armadura se completa con sus cuatro faldones. Los menores aportan al almizate cinco vigas denominadas partorales, dejando el resto de la anchura de la armadura a ocho vigas -ahora denominadas péndolas- que se unen a la que ejerce de vértice entre los faldones, denominada limabordón, sin prolongarse en el almizate, pues mueren en la zona decorada del mismo. Los faldones mayores o gualderas si que se continúan en el almizate, cruzando de lado a lado por detrás de las zonas decoradas del mismo. Todo el entramado se decora mediante perfiles dorados, siendo el almizate la zona más decorada.

El almizate, o zona plana en el centro de la armadura, presenta tres paneles rectangulares configurados por decoración de lazo, integrada por estrellas de ocho puntas, crucetas y aspas. De los tres paneles, los de pies y cabeza son iguales: rectángulos cuyo lados mayores se orientan a los menores de la armadura decorados por lazería a base de doce estrellas de ocho puntas y el mismo número aspas, que se presentan oblicuas respecto al eje longitudinal de la armadura (Fic. 13). El lado mayor del panel lo ocupan tres estrellas y tres aspas, mientras que el menor lo hacen 


Q 2.Vilo Rafael Valentín López Flores

\section{Armadura del \\ Palacio de los Marqueses de \\ Moctezuma; tirante central de la armadura}

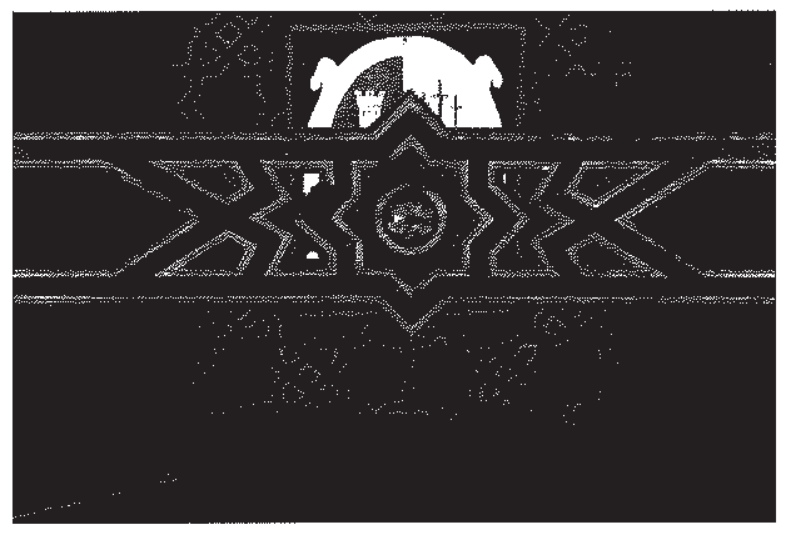

13. Armadura del
Palacio de los
Marqueses de
Moctezuma; uno
de los paneles
menores de
lazería en el
almizate de la
armadura

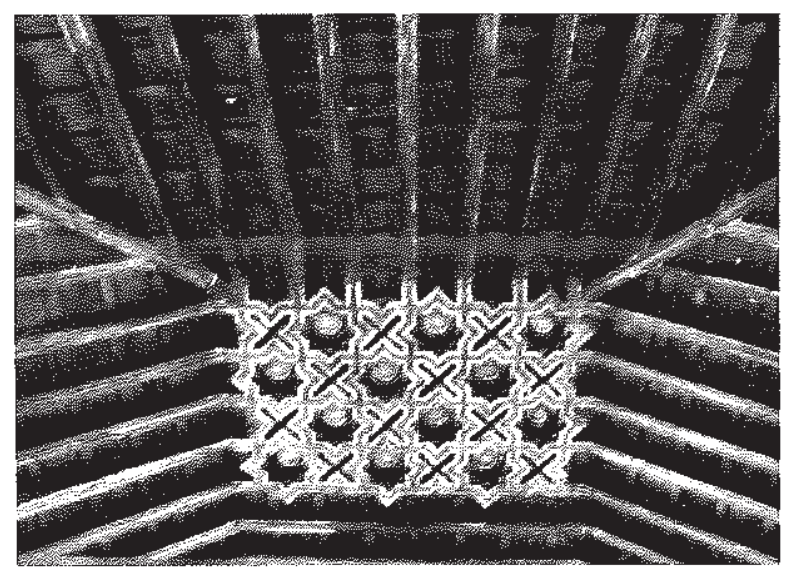

dos y dos; decorando todos sus contornos con las ya conocidas líneas doradas. Tanto las aspas como las estrellas se aderezan en sus perfiles con decoración en forma de puntas de diamante, que el caso de las estrellas serán hacía el exterior, siguiendo los vértices de las puntas de la estrella, y en el caso de las aspas hacia el interior. Las aspas están -como el resto de decoración que hasta ahora hemos vistocaladas, mientras que las estrellas son talladas, generando tabicas octogonales decoradas en su interior por rosetas doradas a modo de soles.

El panel central adopta como lado mayor el propio de la armadura, y deja su centro libre para albergar en él un escudo heráldico (Fic. 14). Por lo demás el perímetro de dicho panel lo conforman una línea de estrellas de ocho puntas iguales a las anteriores, y crucetas que han cambiado su disposición oblicua por la longitudinal. Aparecen de ese modo dos estrellas juntas en el centro, computando -como en los dos paneles menores- doce estrellas y crucetas. Su decoración es muy similar a la de los casos anteriores, repitiendo los perfiles en punta de diamante, las molduras doradas, etc.; sólo la inserción de un escudo heráldico en su centro la diferencia. 


\begin{tabular}{|l} 
14. Armadura del \\
Palacio de los \\
Marqueses de \\
Moctezuma; \\
escudo heráldico \\
en el panel central \\
de lazería del \\
almizate de la \\
armadura
\end{tabular}

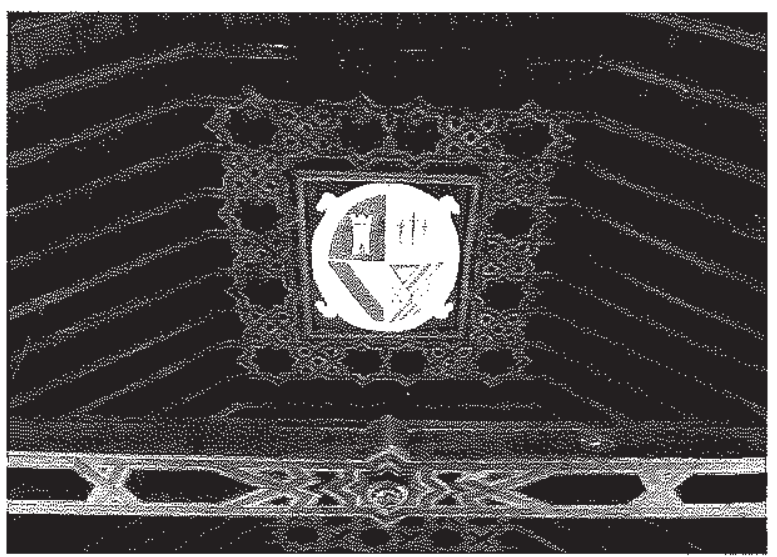

Será el Doctor D. Sebastián García Garrido ${ }^{12}$ quien nos de información relativa al escudo. Se conforma por cuatro cuarteles enmarcados por un óvalo dorado. Tres de ellos representan los emblemas del linaje de los Ovalle. El primero de ellos -ángulo superior izquierdo según la Fig. 14- muestra un castillo de plata en fondo de azur sobre un monte con dos lobos a sus lados, flanqueado por dos árboles atravesados y coronado por una cruz roja flordelisada de gules. En el segundo -ángulo superior derecho- se presentan tres espadas sobre fondo dorado dispuestas en palo con la punta hacia abajo, y el cuarto -ángulo inferior izquierdo- lo compone una banda de azur sobre oro. El cuarto cuartel -ángulo inferior derecho- se deja para el linaje Mendoza a través de un cuartelado en frange a base de sinople, una banda de gules, y dos series de paneles de gules puestas sotuer. Podría corresponder este escudo al primero de los titulares del señorío Ovalle-Dávila y Mendoza -Señores de Arriate- , Rodrigo Ovalle Mendoza, o a su hermana, Isabel de Ovalle -segunda Señora de Arriate-; siendo obra del siglo XIX, cuando este mayorazgo Ovalle recayó en los Moctezuma rondeños.

Terminaremos dando una justificación cronológica a esta armadura. Si nos atenemos a las características del palacio que la alberga o al escudo que la remata, tendríamos que decir que se trata de una armadura del siglo XIX. Pero las directrices general que sigue, su decoración y sus grandes similitudes con otras de la provincia nos hacen adoptar el siglo XVI como base cronológica para su ejecución. La clara estética mudéjar-renacentista de proporcionalidad y simetría, los remates de perfil en $S$ de los canecillos y zapatas de la armadura, las aspas, crucetas y estrellas de ocho puntas con rosetas a modo de soles, típicamente mudéjares, las grandes similitudes con las cercanas armaduras de esa misma centuria en Ardales, Almogía o Almargen,

${ }^{12}$ GARCÍA GARRDDO, Sebastián: El diseño heráldico como lenguaje visual. Heráldica nobiliaria de la ciudad de Ronda, Málaga, Universidad, 1998, págs. 451-454. 
y el conocimiento de que el palacio existía desde los tiempos de la reconquista ustamente a un tal Rodrigo Ovalle-, nos hace pensar estamos ante una armadura del siglo XVI. Respetada en las sucesivas reformas del edificio, y reformada con el añadido o sustitución del escudo que hoy presenta; lo que concuerda con las apreciaciones de Sebastián García Garrido, que vio en la dificultad de ceñirse a un espacio predefinido la causa de los fallos heráldicos que el escudo de la armadura presenta. Todo esto os hace pensar en una armadura que desde el siglo XVI ha sobrevivido a múltiples reformas, llegando a nuestros días haciendo gala de su gran belleza. 